\title{
REVIEWS
}

\section{The Role of HSP70 Heat Shock Proteins in the Pathogenesis and Treatment of Inflammatory Bowel Diseases}

Department of Internal Diseases, Metabolic Diseases and of Dietetics, Clinical Hospital No. 2, Poznan Medical University, Poland

A - research concept and design; $\mathbf{B}$ - collection and/or assembly of data; $\mathbf{C}$ - data analysis and interpretation;

$\mathbf{D}$ - writing the article; $\mathbf{E}$ - critical revision of the article; $\mathbf{F}$ - final approval of article; $\mathbf{G}$ - other

\begin{abstract}
Heat shock proteins (HSPs) represent an important element in the body's defense against various damaging factors. The probably also play an important role in the pathogenesis and treatment of several diseases, including autoimmune pathology and neoplasms. Recently, several investigators have focused their attention on the involvement of the HSP70 protein family in the morbid process of inflammatory bowel diseases (IBD). The HSP70 family of is represented by two distinct forms of protein, the HSP72 protein (also known as the HSP70.1 protein), the expression of which is clearly increased in conditions of stress; and the HSP73 (or HSC73) protein, which manifests stable expression. HSP70 proteins are present in the colorectal epithelium. In patients with inflammatory bowel diseases, their expression in significantly increased during the active stage of the disease. In experimental studies, overexpression of HSP70 was found to prevent the development of inflammatory process in the large intestinal mucosa provoked by various damaging factors. In physiological conditions, various mechanisms are considered to be responsible for an increased expression of HSP70. One of them involves lymphocyte activity and the production of cytokines (mainly IL-2). Another suggested mechanism involves the presence of bacteria in the large intestine, including both physiological flora (Lactobacillus GG, Bacteroides fragilis) and pathogenic bacteria (Salmonella, Escherichia coli). HSP70 expression is probably also increased by physical activity. There is also a potential for pharmacological stimulation of HSP70 expression, linked (for example) to geranylgeranylacetone, polaprezinc and mesalazine. Thus, augmentation of HSP70 expression may become a new element in IBD therapy (Adv Clin Exp Med 2015, 24, 3, 525-530).
\end{abstract}

Key words: heat shock proteins, inflammatory bowel diseases.

Heat-shock proteins (HSPs) are present in all living organisms; their presence has been detected both in simple monocellular species and in highly developed vertebrates, including humans [1]. The presence of HSP-coding genes was described for the first time in 1962 [2], but the proteins themselves were discovered in 1974 in Drosophila melanogaster [3]. The name of heat-shock proteins originated from the observation that their concentration increases following exposure to thermal stress. However, it has been found that HSPs are produced in several other risky situations, such as oxidative stress, exposure to chemical agents, biological agents (viral infections) or physical agents
(UV radiation), a disturbed blood supply or insufficient nutrition [4]. In such situations the proteins protect cells and promote reparative processes in the damaged proteins [5]. Therefore, HSPs are sometimes thought to be chaperone proteins, even though many of them also function as proteases, participating in eliminating the damaged proteins. Recently, increasing attention has been devoted to the role of HSPs in immune processes. In currently ongoing investigations a few HSP receptors have been identified on antigen-presenting cells (APC) and immune processes that follow their activation have been experimentally confirmed [6]. Thus, the pathway of HSP interaction 
with the immune system has been demonstrated, even though the mechanism involved in the interaction still needs to be fully elucidated. HSPs are thought to represent an important element in the body's natural defense against damage due to autoimmune mechanisms. Therefore, investigators focus on the role of HSPs both as an element of pathophysiology in immune processes and as a potential therapeutic target in autoaggressive diseases. In addition, autoaggressive diseases are not the only group of diseases in which the potential for the therapeutic application of HSPs has been considered. In oncology investigations continue on individualized vaccines directed against mutated HSPs that are typical of a given tumor [7]. This method of treatment is defined as an active specific immunotherapy using a non-cellular vaccine [8].

Another interesting theory about the way HSPs may interact with the human immune system involves the unfavorable phenomenon of molecular mimicry. This phenomenon is caused by a structural similarity between HSP molecules present in the cells of pathogenic bacteria and human HSP proteins. This may result in bacterial infectionstimulated production of anti-HSP antibodies, which leads to an autoimmune reaction. Most of the studies focused on this problem are observations of possible anti-self HSP reactivity following Mycobacterium tuberculosis (MT) and Chlamydia trachomatis (ChT) infections. ChT infection causes the formation of antibodies against ChT HSP60, but also against HSP60 of the host infected by the bacteria. These antibodies may become the cause of autoimmune reactions [9]. In MT infection, it is hypothesized that bacterial HSP70 may provoke T-lymphocyte reactivity. T-cell stimulation can be a trigger factor for autoimmune diseases such as rheumatoid arthritis or multiple sclerosis [10-12]. However, there have also been studies that rebut the theory of autoimmunity provocation by the mechanism of molecular mimicry [13].

HSPs are classified into families of differing molecular weights, which is reflected by their nomenclature, e.g. the $70 \mathrm{kDa}$ protein family of is called HSP70. Proteins of a given family may fulfil various functions [5].

Inflammatory bowel diseases (IBDs) represent a group of systemic autoimmune diseases with the involvement of the alimentary tract, including ulcerative colitis, Leśniowski-Crohn's disease, microscopic colitis and undefined colorectal inflammation. A detailed etiology of the autoimmune processes involved has not yet been determined. The treatment of IBDs continues to pose a serious medical challenge. Even though new drugs have appeared in recent years, in many patients the course of these diseases remains difficult to control, worsens the quality of life and leads finally to intestinal resection. In view of the documented chronic unfavorable effects, the surgical approach cannot be treated as the optimum therapeutic strategy; therefore numerous investigations continue to be undertaken in search of new potential pharmacological treatments.

According to recent study results, a few HSP families seem to play a significant role in the pathogenesis of inflammatory bowel diseases [14]. One of them is the HSP70 protein family. It is thought that they exhibit a potential for enhancing a specific natural defense against tissue injury due to an autoimmune mechanism. Therefore, investigations have been undertaken looking for ways to affect the activity of HSP70 proteins as a possible therapeutic strategy. In this paper, current views on role of HSP proteins in the pathophysiology and course of diseases, and in the treatment of inflammatory bowel diseases, have been reviewed.

\section{HSP70 in the Colorectal Mucosa of Patients with Inflammatory Bowel Diseases}

Two groups of HSP proteins with a molecular weight of $70 \mathrm{kDa}$ are distinguished: HSP72 (which used to be abbreviated as HSP70), the concentration of which markedly increases in stress situations, and HSP73 (also known as HSC73, short for heat shock cognate protein), which manifests stable expression regardless of the conditions [14-16]. The decisive majority of studies on the kinetics of expression involving proteins of the HSP70 family thus pertain to HSP72. The expression of HSC73 proteins is similar in the small intestine (jejunum and ileum) and the in large intestine. HSP72, in turn, are manifested almost exclusively in the large intestine (a small degree of HSP72 expression can also be detected in the terminal fragment of the ileum - the so-called ileum terminale). HSP72 concentration is clearly the highest in the mucosa contacting the intestinal lumen, i.e. in cells of the upper portions of intestinal crypts [17].

The specific transcription factor HSF1 (heat shock factor 1) is responsible for the expression of HSP proteins. In stress situations it activates the gene coding for HSP70 proteins. In its absence (e.g., in an experimental model of mice devoid of HSF1) the expected expression of HSP70 is absent [18]. Potential HSP70 expression is also determined by genes. There is a specific genotype (HSP70-2 BB) that is associated with a better prognosis for the course of ulcerative colitis; however, 
it is not related to the risk of developing the disease [19].

In inflammatory diseases HSP70 expression is significantly higher than in a healthy population. In studies performed on animals, increased expression of HSP70 proteins and HSP40 proteins was detected in the colorectal mucosa of mice with pharmacologically induced colitis. Interestingly, not all HSP families were found to undergo similar processes - the expression of HSP25, HSP32 and HSP90 remained unchanged [20]. Similar observations were found in studies involving human populations, in which increased HSP70 expression was detected in the mucosa of patients with Crohn's disease and ulcerative colitis, as compared to the mucosa of healthy volunteers. The evaluation included not only biopsies of mucosa with macroscopically evident pathology, but also fragments described as intact in endoscopic examination: in both cases HSP70 expression was higher than in the control group [21].

High HSP70 expression is typical for the active phase of inflammatory bowel diseases, and it undergoes a decrease upon their effective treatment. In a population of patients with ulcerative colitis, HSP70 expression in the mucosa was markedly higher prior to six months of therapy with 5-ASA preparations and probiotics. This observation suggest that following remission, HSP levels in patients with inflammatory bowel diseases do not differ from those in the healthy population [22].

On the other hand, antibiotic treatment for other reason eradicates commensal bacteria and can decrease HSP70 expression. Probably some bacterial epitopes are necessarily for proper HSP70 induction [23].

Characteristic changes in HSP70 expression are observed in the epithelium of colorectal mucosa depending on the presence of the inflammatory process and on its activity (Fig. 1). In contrast, in the lamina propria the expression of HSP proteins is much lower and seems to be stable, independently of the presence of inflammatory lesions [22].

$$
\text { IBD - active phase }
$$

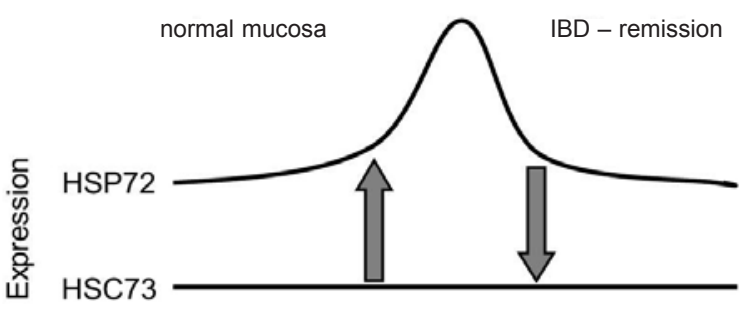

Fig. 1. The expression of HSP proteins in relation to inflammation in the large intestinal mucosa. IBD - inflammatory bowel disease
To summarize, according to recent studies, HSP70 expression in the colorectal mucosa of IBD patients depends on various mechanisms. In general, the possible increase in HSP levels is determined by genes and intestinal bacterial flora. In the active phase of ulcerative colitis HSP70 expression is higher; it is also higher in visibly inflamed mucosa than in macroscopically unchanged mucosa. When remission is achieved due to intensification of drug treatment, HSP70 expression decreases to a level close to or even equal to the level found in the healthy population.

\section{The Role of HSP70 in the Pathological Process of Inflammatory Bowel Diseases}

The presence of HSP70 proteins in augmented amounts may be a factor that inhibits the development of the inflammatory process in colorectal mucosa. This conclusion can be drawn from an experiment conducted on mice devoid of the genes that code for macrophage migration inhibitory factor. This mutation resulted in a marked augmentation of HSP70 and HSP40 in the intestinal mucosa of the experimental animals; this in turn inhibited the development of pharmacologically induced colorectal inflammation. Nevertheless, after the administration of a pharmacological factor capable of inhibiting HSP expression, the animals developed colorectal inflammation. This proved that it was just the augmented expression of HSP which protected the mice from the development of the disease process [20]. Similar conclusions can be drawn from observations of mice with hyperthermia-induced augmented expression of HSP70 and HSP90. This condition also protected the mice against pharmacologically induced colitis; animals not earlier subjected to hyperthermia developed the disease [24].

Despite the increase of HSP70 expression as a typical response to mucosa damaging factors, some basic expression of HSP70 can be detected in the colonic wall.

It is suspected that HSP70 expression is stimulated by the cells of the immune system. In an experiment conducted on mice genetically deprived of lymphocytes T and B, HSC73 expression was the same as in mice with the unmodified genome; expression of HSP70, in turn, was significantly lower in the mice devoid of the lymphocytes. Lymphocytes probably exert the detected effect through the production of cytokines, and interleukin-2 (IL-2) in particular. HSP protein induction was found 
to develop under the influence of other cytokines (IL-10, IL-11, IL-1 $\beta$, TNF- $\alpha$ ), but in this case it involved mainly the HSP25 family, with no effect on HSP72 and HSC73 [17, 25]. A reciprocal relation also exists between mediators of inflammatory conditions and HSP70. In a pharmacologically induced model of colorectal inflammation, in mice with augmented HSP70 expression a significantly lower expression was observed of pro-inflammatory cytokines, such as TNF- $\alpha$, IL-6, IL- $1 \beta$ as well as lower macrophage activity, as compared to the control group. At the same time, the course of the morbid process was much milder in the mice with augmented HSP70 expression [26]. HSP70 proteins also manifest an ability to stimulate the production of IL-10, which has been found to exert an anti-inflammatory effect in an experimental model of bacterial infection with Listeria monocytogenes and in experimentally induced arthritis [1].

Another variable which significantly increases expression of HSP in cells of the colorectal epithelium is the physiological bacterial flora present in the intestinal lumen. Among others, soluble particles of Lactobacillus GG manifest HSP70 expression stimulating activity [27]. A similar effect is exerted by Bacteroides fragilis strains. No such stimulation was demonstrated in the case of HSC73 molecules, the expression of which seemed to be independent of the presence of individual bacterial strains in the intestinal lumen [17]. The phenomenon of a low but detectable expression of HSP70 in the terminal fragment of the small intestine may be linked to penetration of the region by large intestinal bacterial flora: It has been proved that even the epithelium of proximal small intestine fragments reacts to contact with colonic bacterial flora by increasing HSP70 expression and by gaining the protective capacity against stress factors that is typical of the presence of these proteins [28]. A similar relationship has also been proved for pathogenic bacteria such as Salmonella enteritidis or Escherichia coli $[29,30]$. A relationship between HSP70 expression and the bacterial flora of the intestinal lumen was also confirmed by the phenomenon of decreasing expression of these proteins following treatment with metronidazole (a chemotherapeutic agent particularly active against anaerobes). No such relationship could be demonstrated for ciprofloxacin, another widely-used antibiotic $[17,28]$.

Another probable physiological variable capable of increasing HSP70 expression is physical activity [31]. This may be one of the mechanisms by which physical exertion favorably affects the clinical course of inflammatory processes, including nonspecific intestinal inflammation (Fig. 2). To date, the hypothesis has not been proved experimentally, but studies have already been published in

\section{Physical effort}

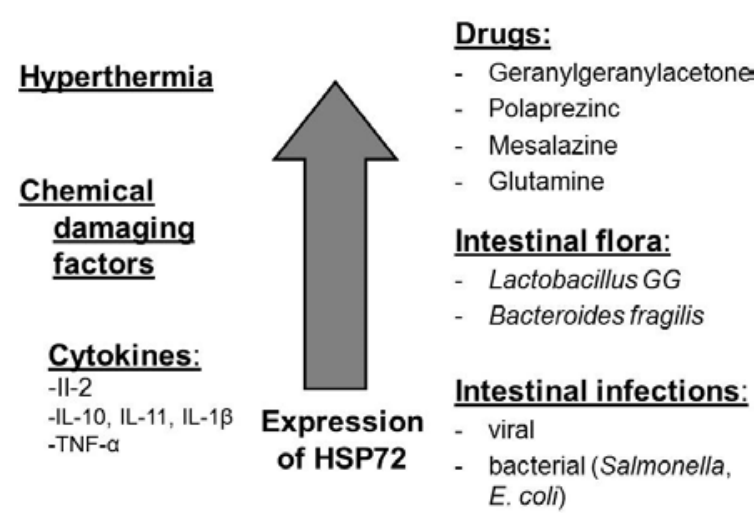

Fig. 2. Factors increasing HSP72 expression in the large intestinal mucosa

which an increase in HSP70 expression developed in the heart and skeletal muscles as an effect of physical exertion $[32,33]$. This results in a favorable, cardio-protective phenomenon [34].

\section{Potential Pharmacological Approaches}

The evident relationship between expression of HSP70 proteins in the intestinal epithelium and a protective ability against damaging factors opens new therapeutic perspectives in intestinal diseases. In line with the observations described above, pharmacological stimulation of HSP70 expression might favorably affect the course of colorectal bowel disease or even prevent its development. Pharmacological stimulation of HSP70 expression is manifested by geranylgeranylacetone, a substance of experimentally proven ability to augment HSP70 expression in the intestinal mucosa. This ability results in a milder course or prevention against the development of chemically induced colorectal inflammation $[35,36]$. Polaprezinc is another substance that stimulates HSP70 expression. This chemical compound of zinc and L-carnosine manifests anti-neoplastic and anti-oxidative properties. Administered at an appropriate time before a chemical insult to the large intestine, it can prevent the development of an inflammatory condition. At the same time, a marked increase in the expression of HSP70 is also noted [37]. In addition, the administration of quercetin (a substance that inhibits HSP70 expression) has been found to ablate the anti-inflammatory action of polaprezinc [38]. The protective action of polaprezinc has also been detected in a similar mechanism in cases of exposure of the small intestine to injury due to acetylsalicylic acid [39]. 
Mesalazine is one of the drugs that have been used for years in treating inflammatory bowel diseases. The substance also affects HSP70 expression, although in an indirect way. Administration of mesalazine itself fails to increase HSP70 expression in the cells of the colorectal mucosa. However, when administration of the drug is followed by exposure to hyperthermia, mesalazine potentiates the effect of high temperature, additionally increasing HSP70 expression [40]. It thus allows a better protective effect against oxidative stress to be obtained.

Glutamine administered in high doses also has exerts a protective effect in experimental large intestine inflammation. The mechanism of the protective action is probably based on increased concentrations of HSP70 and HSP25 proteins, which are observed after the substance is administered [41].

\section{Summary}

Heat shock proteins, including the HSP70 family, certainly constitute an important element in the pathophysiology of inflammatory bowel diseases. Their expression clearly increases when cell damaging factors appear, including those which accompany the inflammatory process. HSP70 proteins provide a protective effect for colorectal mucosa and therefore restrict the morbid process. Due to the latter effect, increased HSP70 expression in the intestinal mucosa represents a potential therapeutic target which may constitute an important alternative or supplementation of current approaches to inflammatory bowel diseases.

\section{References}

[1] Borges TJ, Wieten L, van Herwijnen MJ, Broere F, van der Zee R, Bonorino C, van Eden W: The anti-inflammatory mechanisms of HSP70. Front Immunol 2012, 3, 95.

[2] Ritossa FA: A new puffing pattern induced by temperature shock and DNP in Drosophila. Experientia 1962, 18, 571-573.

[3] Tissières A, Mitchell HK, Tracy U: Protein synthesis in salivary glands of Drosophila melanogaster: relation to chromosome puffs. J Mol Biol 1974, 84, 389-398.

[4] Lindquist S: The heat-shock response. Annu Rev Biochem 1986, 55, 1151-1191.

[5] Pockley AG: Heat shock proteins as regulators of the immune response. Lancet 2003, 362, 469-476.

[6] Binder RJ, Vatner R, Srivastava P: The heat-shock protein receptors: some answers and more questions. Tissue Antigens 2004, 64, 442-451.

[7] Kowalczyk DW: Therapeutic vaccines in carcinoma of the kidney (in Polish). Współczesna Onkol 2007, 11, 160-166.

[8] Mackiewicz J, Mackiewicz A: Immunotherapy of tumours and perspectives for its development (in Polish). Współczesna Onkol 2010, 14, 59-71.

[9] Cappello F, Conway de Macario E, Di Felice V, Zummo G, Macario AJ: Chlamydia trachomatis infection and anti-HSP60 immunity: the two sides of the coin. PLoS Pathog 2009, Aug, 58.

[10] Res PC, Schaar CG, Breedveld FC: Synovial fluid T cell reactivity against $65 \mathrm{kD}$ heat shock protein of mycobacteria in early chronic arthritis. Lancet 1988, 2, 478-480.

[11] Salvetti M, Buttinelli C, Ristori G: T-lymphocyte reactivity to the recombinant mycobacterial $65-$ and $70-\mathrm{kDa}$ heat shock proteins in multiple sclerosis. J Autoimmun 1992, 5, 691-702.

[12] Chodisetti SB, Rai PK, Gowthaman U: Potential T cell epitopes of Mycobacterium tuberculosis that can instigate molecular mimicry against host: implications in autoimmune pathogenesis. BMC Immunol 2012, 13, 13.

[13] Van Eden W, Thole J, van der Zee R: Cloning of the mycobacterial epitope recognized by T lymphocytes in adjuvant arthritis. Nature 1988, 331, 171-173.

[14] Otaka M, Odashima M, Watanabe S: Role of heat shock proteins (molecular chaperones) in intestinal mucosal protection. Biochem Biophys Res Commun 2006, 348, 1-5.

[15] Itoh H, Tashima Y: The stress (heat shock) proteins. Int J Biochem 1991, 23, 1185-1191.

[16] Lindquist S: Varying patterns of protein synthesis in Drosophila during heat shock: implications for regulation. Dev Biol 1980, 77, 463-479.

[17] Kojima K, Musch MW, Ren H, Boone DL, Hendrickson BA, Ma A, Chang EB: Enteric flora and lymphocytederived cytokines determine expression of heat shock proteins in mouse colonic epithelial cells. Gastroenterology 2003, 124, 1395-1407.

[18] Tanaka K, Mizushima T: Protective role of HSF1 and HSP70 against gastrointestinal diseases. Int J Hyperthermia 2009, 25, 668-676.

[19] Tahara T, Shibata T, Okubo M, Ishizuka T, Kawamura T, Yamashita H, Nakamura M, Nakagawa Y, Nagasaka M, Arisawa T, Ohmiya N, Hirata I: Heat-shock protein 70-2 BB genotype is associated with reduced risks of the steroid-dependent and refractory phenotypes of ulcerative colitis. Biomed Rep 2014, 2, 555-558.

[20] Ohkawara T, Nishihira J, Ishiguro Y, Otsubo E, Nagai K, Takeda H, Kato M, Yoshiki T, Iwanaga T, Asaka M: Resistance to experimental colitis depends on cytoprotective heat shock proteins in macrophage migration inhibitory factor null mice. Immunol Lett 2006, 107, 148-154. Epub 2006, Sep 26.

[21] Ludwig D, Stahl M, Ibrahim ET, Wenzel BE, Drabicki D, Wecke A, Fellermann K, Stange EF: Enhanced intestinal expression of heat shock protein 70 in patients with inflammatory bowel diseases. Dig Dis Sci 1999, 44, $1440-1447$. 
[22] Tomasello G, Sciumé C, Rappa F, Rodolico V, Zerilli M, Martorana A, Cicero G, De Luca R, Damiani P, Accardo FM, Romeo M, Farina F, Bonaventura G, Modica G, Zummo G, Conway de Macario E, Macario AJ, Cappello F: HSP10, HSP70, and HSP90 immunohistochemical levels change in ulcerative colitis after therapy. Eur J Histochem 2011, 55, 38.

[23] Matsuo K, Zhang X, Ono Y, Nagatomi R: Acute stress-induced colonic tissue HSP70 expression requires commensal bacterial components and intrinsic glucocorticoid. Brain Behav Immun 2009, 23, 108-115.

[24] Otani S, Otaka M, Jin M, Okuyama A, Itoh S, Iwabuchi A, Sasahara H, Itoh H, Tashima Y, Masamune O: Effect of preinduction of heat shock proteins on acetic acid-induced colitis in rats. Dig Dis Sci 1997, 42, 833-846.

[25] Ropeleski MJ, Tang J, Walsh-Reitz MM, Musch MW, Chang EB: Interleukin-11-induced heat shock protein 25 confers intestinal epithelial-specific cytoprotection from oxidant stress. Gastroenterology 2003, 124, 1358-1368.

[26] Tanaka K, Namba T, Arai Y, Fujimoto M, Adachi H, Sobue G, Takeuchi K, Nakai A, Mizushima T: Genetic evidence for a protective role for heat shock factor 1 and heat shock protein 70 against colitis. J Biol Chem 2007, 282, 23240-23252.

[27] Tao Y, Drabik KA, Waypa TS, Musch MW, Alverdy JC, Schneewind O, Chang EB, Petrof EO: Soluble factors from Lactobacillus GG activate MAPKs and induce cytoprotective heat shock proteins in intestinal epithelial cells. Am J Physiol Cell Physiol 2006, 290, 1018-1030.

[28] Arvans DL, Vavricka SR, Ren H, Musch MW, Kang L, Rocha FG, Lucioni A, Turner JR, Alverdy J, Chang EB: Luminal bacterial flora determines physiological expression of intestinal epithelial cytoprotective heat shock proteins 25 and 72. Am J Physiol Gastrointest Liver Physiol 2005, 288, 696-704.

[29] Malago JJ, Koninkx JF, Ovelgönne HH, van Asten FJ, Swennenhuis JF, van Dijk JE: Expression levels of heat shock proteins in enterocyte-like Caco-2 cells after exposure to Salmonella enteritidis. Cell Stress Chaperones 2003, 8, $194-203$.

[30] Deitch E, Beck S, Cruz N, De Maio A: Induction of heat shock gene expression in colonic epithelial cells after incubation with Escherichia coli or endotoxin. Crit Care Med 1995, 23, 1371-1376.

[31] Chen Y, Noble EG: Is exercise beneficial to the inflammatory bowel diseases? An implication of heat shock proteins. Med Hypotheses 2009, 72, 84-86.

[32] Melling CW, Thorp DB, Noble EG: Regulation of myocardial heat shock protein 70 gene expression following exercise. J Mol Cell Cardiol 2004, 37, 847-855.

[33] Noble EG, Ho R, Dzialoszynski T: Exercise is the primary factor associated with HSP70 induction in muscle of treadmill running rats. Acta Physiol (Oxf) 2006, 187, 495-501.

[34] Paroo Z, Haist JV, Karmazyn M, Noble EG: Exercise improves postischemic cardiac function in males but not females: consequences of a novel sex-specific heat shock protein 70 response. Circ Res 2002, 90, 911-917.

[35] Ohkawara T, Nishihira J, Takeda H, Katsurada T, Kato K, Yoshiki T, Sugiyama T, Asaka M: Protective effect of geranylgeranylacetone on trinitrobenzene sulfonic acid-induced colitis in mice. Int J Mol Med 2006, 17, $229-234$.

[36] Ohkawara T, Takeda H, Nishiwaki M, Nishihira J, Asaka M: Protective effects of heat shock protein 70 induced by geranylgeranylacetone on oxidative injury in rat intestinal epithelial cells. Scand J Gastroenterol 2006, 41, 312-317.

[37] Ohkawara T, Takeda H, Kato K, Miyashita K, Kato M, Iwanaga T, Asaka M: Polaprezinc (N-(3-aminopropionyl)L-histidinato zinc) ameliorates dextran sulfate sodium-induced colitis in mice. Scand J Gastroenterol 2005, 40, 1321-1327.

[38] Ohkawara T, Nishihira J, Nagashima R, Takeda H, Asaka M: Polaprezinc protects human colon cells from oxidative injury induced by hydrogen peroxide: relevant to cytoprotective heat shock proteins. World J Gastroenterol 2006, 12, 6178-6181.

[39] Qin Y, Naito Y, Handa O: Heat shock protein 70-dependent protective effect of polaprezinc on acetylsalicylic acid-induced apoptosis of rat intestinal epithelial cells. J Clin Biochem Nutr 2011, 49, 174-181. DOI: 10.3164/ /jcbn.11-26. Epub 2011 Jun 17.

[40] Burress GC, Musch MW, Jurivich DA: Effects of mesalamine on the HSP72 stress response in rat IEC-18 intestinal epithelial cells. Gastroenterology 1997, 113, 1474-1479.

[41] Xue H, Sufit A, Wischmeyer P: Glutamine Therapy Improves Outcome of In Vitro and In Vivo Experimental Colitis Models JPEN J Parenter Enteral Nutr 2011, 35, 188-197.

\section{Address for correspondence:}

Paweł Samborski

Department of Internal Diseases, Metabolic Diseases and of Dietetics

Clinical Hospital No. 2

Poznan Medical University

Przybyszewskiego 49

60-355 Poznań

Poland

Tel.:+48 660470101

E-mail: samborski.pawel.mail@gmail.com

Conflict of interest: None declared 